\title{
Transcutaneous application of oil and prevention of essential fatty acid deficiency in preterm infants
}

Department of

Paediatrics and

Child Health,

Flinders Medical

Centre,

Bedford Park, South

Australia 5042

Emma J Lee

Robert A Gibson

Karen Simmer

Correspondence to: Dr Simmer.

Accepted 30 June 1992

\begin{abstract}
The topical application of vegetable oil was assessed as an alternative means of providing essential fatty acids (EFA) to parentally fed preterm infants who were not receiving lipid. Three infant pairs ranging in gestational age from 26-32 weeks were studied. Safflower oil or safflower oil esters ( $1 \mathrm{~g}$ linoleic acid/kg/day) were applied to available areas daily. All infants rapidly developed biochemical EFA deficiency. The plasma fatty acid profiles were similar in infants with or without topical oil, and all returned to normal once parenteral lipid was introduced. We found no evidence to suggest that the transdermal route is of use in the nutritional management of preterm infants.
\end{abstract}

\section{(Arch Dis Child 1993;68:27-8)}

Parenterally fed preterm infants rapidly become deficient in essential fatty acids (EFA) unless lipid is administered. Lipid emulsions, however, are often delayed or omitted because of their association with hypoxia and chronic lung disease, ${ }^{1-3}$ and concern over interference with bilirubin binding. ${ }^{4}$

Our aim was to assess whether the topical application of a polyunsaturated vegetable oil could provide an alternative means of providing EFA. The transcutaneous route seemed a possibility as the preterm neonate has very permeable skin ${ }^{5}$ and drugs have been successfully administered to neonates by this means. ${ }^{6}$

\section{Patients and methods}

Infants of less than 2 weeks of age were studied, as drug absorption correlates well with epidermal structural maturation and by 2 weeks of age the rate of absorption is reduced to that of term infants. ${ }^{5}$ Three infant pairs were studied: (A) <1000 g, <28 weeks' gestation; (B) 1000-1500 g, 28-30 weeks' gestation; and (C) $>1500 \mathrm{~g},>30$ and $<34$ weeks' gestation.

Birth weight, gestational age, and topical oil treatment of three matched pairs of premature infants

\begin{tabular}{rlllc}
\hline Pair & $\begin{array}{l}\text { Birth weight } \\
(\mathrm{g})\end{array}$ & $\begin{array}{l}\text { Gestational age } \\
\text { (weeks) }\end{array}$ & Days oil applied & $\begin{array}{l}\text { Day IV lipid } \\
\text { introduced }\end{array}$ \\
\hline (A) 1 & 732 & 26 & 8 & 11 \\
2 & 955 & 26 & 0 & 9 \\
(B) 1 & 1310 & 30 & $5(6-10)$ & 10 \\
2 & 1140 & 29 & 0 & 6 \\
(C) 1 & 1740 & 33 & 4 & 5 \\
2 & 1874 & 31 & 0 & 5 \\
\hline
\end{tabular}

IV, intravenous.
All infants were receiving total parenteral nutrition. Parenteral lipid was prescribed by the clinician when the infant was stable and jaundice had resolved. This varied from day 4-10 and was independent of the investigation.

Oil was applied to three infants while three others, who were matched for height, weight and days of fat-free alimentation, acted as controls. Safflower oil (Crisco Foods, Australia) was used for pairs (A) and (B), and safflower esters (Technu Laboratories, Australia) for pair (C). The esters were used to determine whether this form was better absorbed than the triglyceride (oil) form. The oil and esters contained $78 \%$ linoleic acid, $10 \%$ saturates and $12 \%$ monounsaturates. Vitamin $\mathrm{E}$ was added at $1600 \mathrm{IU} / 100$ $\mathrm{ml}$ and all oils were stored at $4^{\circ} \mathrm{C}$ in the dark.

A small pilot study determined that the oil was not degraded by phototherapy (less than $1 \%$ change in the fatty acid composition over five hours). Similarly, another small study confirmed that there was no burning of preterm skin under lights and heaters after oil application.

The infant's gestational age and birth weight, the number of days that oil was applied and the day parenteral lipid was commenced are given in the table. The mean daily dose of linoleic acid was $1 \cdot 1$ (range $0.7-2 \cdot 2) \mathrm{g} / \mathrm{kg} /$ day for (A), 0.9 $(0.5-1 \cdot 3) \mathrm{g} / \mathrm{kg} /$ day for $(B)$, and $1.0(0.6-1 \cdot 3)$ $\mathrm{g} / \mathrm{kg} /$ day for (C). Oil was gently massaged into available areas which included the head, upper arms, legs, and buttocks, over 3-4 minutes. Rubber gloves were worn when applying the oil to reduce absorption through the hands.

A total of $200 \mu \mathrm{l}$ of heparinised blood was collected from arterial lines or from heel prick for measurement of linoleic acid and eicosatrienoic acid the endogenously produced fatty acid. Results are expressed as percentage of the total fatty acids. EFA deficiency is defined as linoleic acid concentrations below, and eicosatrienoic acid concentrations above, the normal range. Blood was collected, daily when possible, depending on whether it was being taken for routine tests. The nutritional management and fatty acid analysis methods have been described previously. ${ }^{7}$ The experimental protocol was approved by the Committee on Clinical Investigations at Flinders Medical Centre, and informed parental consent was obtained. 
(A)

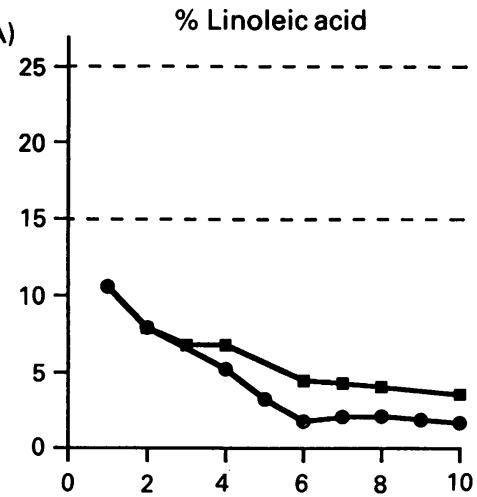

(B)

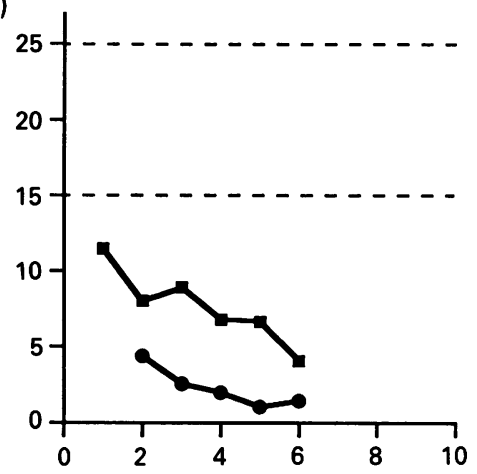

(C)

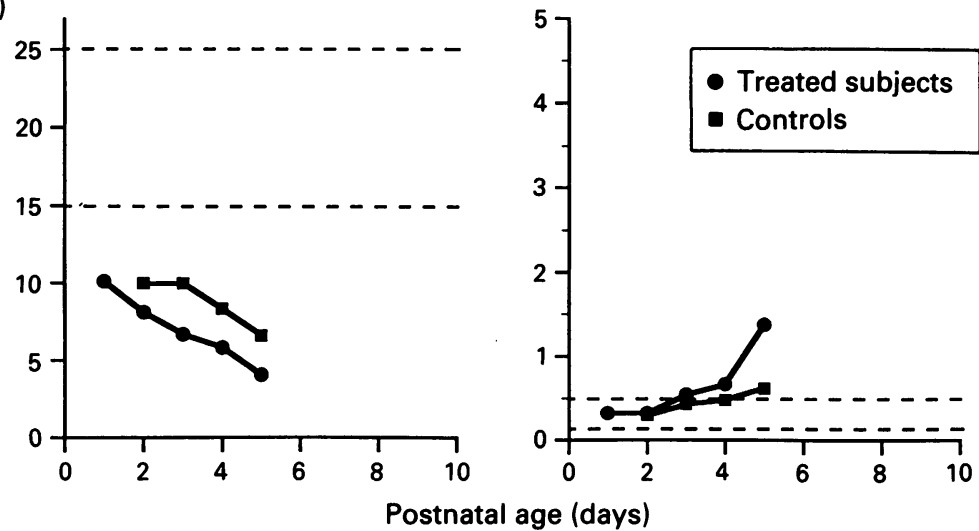

Linoleic acid and eicosatrienoic acid concentrations in plasma of three pairs of parentally fed preterm infants before the introduction of parenteral lipid. $(A)=$ birth weight $<1000 \mathrm{~g}$, $(B)=$ birth weight $1000-1500 \mathrm{~g},(C)=$ birth weight $>1500 \mathrm{~g}$. Topical oil was applied to the treated group. The dotted lines represent the range of plasma fatty acid values found in 11 healthy preterm infants fed enterally from birth with at least $50 \%$ of energy coming from breast milk.
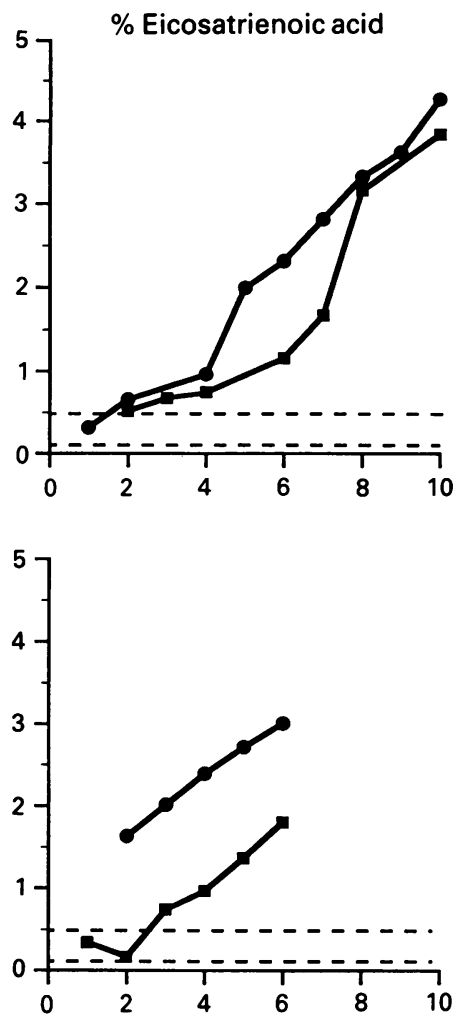

cutaneous administration of EFA, either as triglyceride or as ethyl esters, has a role in the nutritional management of preterm infants. The physiochemical properties of these oils, which are non-polar and thus only lipid soluble, are not conducive to transdermal transfer. Agents possessing both water and lipid solubilities may be more favourably absorbed, ${ }^{13}$ but the difficulties of the limited area available for oil application owing to the presence of many monitors and catheters, in addition to the disturbance to the unstable infant by handling, makes the success of such an approach unlikely.

We gratefully acknowledge the technical assistance of Mark Neumann and the cooperation of the nursing staff of the Neonatal Intensive Care Unit of Flinders Medical Centre.

1 Pereira GR, Fox WW, Stanley CA, Baker L, Schwartz JG. Decreased oxygenation and hyperlipidaemia during intravenous fat infusions in premature infants. Pediatrics 1980 66:26-30.

2 Hammerman C, Aramburo MJ. Decreased lipid intake reduces morbidity in sick premature neonates. $\mathcal{f}$ Pediat reduces morbidity

3 Cooke RWI. Factors associated with chronic lung disease in preterm infants. Arch Dis Child 1991;66:776-9.

4 Andrew G, Chan G, Schiff C. Lipid metabolism in the neonate. II. The effect of Intralipid on bilirubin binding in vitro and in vivo. $\mathcal{F}$ Pediatr 1976;88:279-84.

5 Harpin VA, Rutter N. Barrier properties of the newborn infants skin. F Pediatr 1983;102:419-25.

6 Rutter N. Percutaneous drug absorption. Clin Perinatol 1987;14:911-30.

7 Lee EJ, Simmer KS, Gibson RA. Essential fatty acid deficiency in parenterally fed preterm infants. $₹$ Paediatr Child Health 1992 (in press)

8 Hunt CE, Engel RR, Modler S, Hamilton W, Bissen S, Holman RT. Essential fatty acid deficiency in neonates: inability to reverse deficiency by the topical application of EFA-rich oil. F Pediatr 1978;92:603-7.

9 Friedman Z, Shochat SJ, Maisels J, Marks KH, Lamberth EL. Correction of essential fatty acid deficiency in newborn infants by cutaneous application of sunflower-seed oil. Pediatrics 1976;58:650-4.

10 Vandenplas Y, Leyssens L, Bougatef A, Sacre L, Francois BF. Fatty acid patterns in parenterally fed premature and term infants: changes induced by Intralipid and sunflower seed oil. Am f Perinatal 1989;6:393-6.

11 Postum R Pease PW, Watts R, Taylor S, McEvoy FA. Essential fatty acid deficiency in infants receiving Essential fatty acid deficiency in infants recei

2 Bougle D, Pepin D, Delhaye M, Chambaz J, Ricour C Plasma and erythrocyte essential fatty acids during total parenteral nutrition in infants: effects of cutaneous supparenteral nutrition in infants: effects of cutaneous supply. Four

13 Chien YW. Transdermal control-released drug administration. Novel drug delivery systems-fundamentals, develop ment, concepts and biomedical assessment. New York: Marcel Dekker, 1982;149-217. 